\title{
UNIVERSAL CELL-LIKE MAPS
}

\author{
JERZY DYDAK AND JERZY MOGILSKI
}

(Communicated by James West)

\begin{abstract}
The main results of the paper are the following:
Theorem. Suppose $n \leq \infty$. There is a cell-like map $f: X \rightarrow Y$ of complete and separable metric spaces such that $\operatorname{dim} X \leq n$, and for any cell-like map $f^{\prime}: X^{\prime} \rightarrow Y^{\prime}$ of (complete) separable metric spaces with $\operatorname{dim} X^{\prime} \leq n$ there exist (closed) embeddings $i: Y^{\prime} \rightarrow Y$ and $j: X^{\prime} \rightarrow f^{-1}\left(i\left(Y^{\prime}\right)\right)$ such that $f j=i f^{\prime}$

Corollary. Suppose $n<\infty$. There is a complete and separable metric space $Y$ such that $\operatorname{dim}_{\mathrm{Z}} Y \leq n$, and any (complete) separable metric space $Y^{\prime}$ with $\operatorname{dim}_{\mathbf{Z}} Y^{\prime} \leq n$ embeds as a (closed) subset of $Y$.
\end{abstract}

\section{INTRODUCTION}

This paper is devoted to the existence of universal separable spaces for integral cohomological dimension. The question of existence of such spaces was posed by L. Rubin [Ru] (see also [We]). Rubin's question was generalized in $\left[\mathrm{Dy}_{1}\right]$ :

1.1. Conjecture. If $K$ is a countable $C W$ complex, then there is a universal space in the class of separable metric spaces

$$
\{X \mid K \in A E(X)\} .
$$

Recall that $K \in A E(X)$ means that any map $g: C \rightarrow K, C$ closed in $X$, extends over $X$ (see [Hu]). Also, $S^{n} \in A E(X)$ is equivalent to $\operatorname{dim} X \leq n$, and $K(\mathbf{Z}, n) \in A E(X)$ is equivalent to $\operatorname{dim}_{\mathbf{Z}} X \leq n$ (see [Wa]).

Let us recall work done up to now which relates to Conjecture 1.1.

First, A. Chigogidze [Ch] proved the following result:

1.2. Theorem (A. Chigogidze). Let $n \geq 0$ and $G$ be an abelian group such that there exists a separable completely metrizable ANR-space homotopy equivalent to the Eilenberg-Mac Lane complex $K(G, n)$. Then, there exists a separa-

Received by the editors February 26, 1993.

1991 Mathematics Subject Classification. Primary 55M11, 54F45.

Key words and phrases. Dimension, cohomological dimension, absolute extensors, universal spaces, compactifications, cell-like maps.

Supported in part by a grant from the National Science Foundation. 
ble, completely metrizable space $X(G, n)$ such that the following conditions are equivalent for each metrizable compactum $K$ :

(i) $\operatorname{dim}_{G} K \leq n$,

(ii) $K$ embeds into $X(G, n)$.

Second, Conjecture 1.1 was verified $\left[\mathrm{Dy}_{1}\right]$ for finite complexes:

1.3. Theorem. Suppose $\left\{K_{i}\right\}_{i \geq 1}$ is a sequence of $C W$ complexes homotopy dominated by finite $C W$ complexes. Then

(a) Given a separable, metrizable space $Y$ such that $K_{i} \in A E(Y), i \geq 1$, there exists a metrizable compactification $c(Y)$ of $Y$ such that $K_{i} \in A E(c(Y))$, $i \geq 1$.

(b) There is a universal space of the class

$$
\mathscr{C}=\left\{Y \text { is compact metrizable } \mid K_{i} \in A E(Y) \text { for all } i \geq 1\right\} .
$$

(c) There is a completely metrizable and separable space $Z$ such that $K_{i} \in$ $A E(Z)$ for all $i \geq 1$ with the property that any completely metrizable and separable space $Z^{\prime}$ with $K_{i} \in A E\left(Z^{\prime}\right)$ for all $i \geq 1$ embeds in $Z$ as a closed subset.

Our goal is to prove the existence of universal cell-like maps whose domain are $n$-dimensional. The positive answer to Rubin's question is obtained with the help of the following result:

1.4. Theorem (Rubin-Schapiro [R-S]). If $\operatorname{dim}_{\mathrm{Z}} X \leq n$ and $X$ is a separable metrizable space, then there is a cell-like map $f: X^{\prime} \rightarrow X$ such that $\operatorname{dim} X^{\prime} \leq n$ and $X^{\prime}$ is a separable metrizable space.

Thus, the purpose of this paper is to verify Conjecture 1.1 for EilenbergMacLane spaces $K(Z, n)$. Notice that our techniques can be used to show the existence of universal $\mathbf{Z} / p$-acyclic maps whose domain is $n$-dimensional. Thus, in order to verify Conjecture 1.1 for $K(\mathbf{Z} / p, n)$, all that is needed is to prove the analog of the Rubin-Schapiro Theorem for $Z / p$-coefficients. Such a result in the compact case is due to A. Dranishnikov [ $\left.\mathrm{Dr}_{3}\right]$.

Theorem 1.3 (and its proof) indicated that there ought to be a connection between existence of compactifications in the class $\{X \mid X$ is separable and $K \in$ $A E(X)\}$ and the existence of universal spaces for $\{X \mid X$ is compact and $K \in$ $A E(X)\}$. Such a connection exists:

1.5. Theorem. Suppose $K$ is a $C W$ complex such that any metrizable compactification $c(X)$ of a separable space $X$ with $K \in A E(X)$ admits a metrizable compactification $c^{\prime}(X) \geq c(X)$ (i.e., there is a map $\left.h: c^{\prime}(X) \rightarrow c(X), h \mid X=\mathrm{id}\right)$ with $K \in A E\left(c^{\prime}(X)\right)$. Then, there is a map $\pi: M \rightarrow Q$ of a compactum $M$ to the Hilbert cube $Q$ such that $K \in A E(M)$ and for any map $g: X \rightarrow Q, X$ separable metrizable, and $K \in A E(X)$, there is an embedding $i: X \rightarrow M$ with $\pi i=g$.

Thus, Theorem 1.3 is a consequence of Theorem 1.5 and a result of Shvedov (use $K$ being the weak product $\lim \prod_{i=1}^{n} K_{i}$ of $K_{i}, i \geq 1$ ):

1.6. Theorem (I. A. Shvedov [Ku]). Let $X$ be a separable and metrizable space and suppose $K_{i} \in A E(X), i \geq 1$, are $C W$ complexes homotopy dominated by finite polyhedra. Then, for any metrizable compactification $c(X)$ of $X$, there 
exists a metrizable compactification $c^{\prime}(X) \geq c(X)$ (i.e., there is a map $h$ : $c^{\prime}(X) \rightarrow c(X)$ with $\left.h \mid X=\mathrm{id}\right)$ of $X$ such that $K_{i} \in A E\left(c^{\prime}(X)\right)$ for all $i \geq 1$.

Remark. Strictly speaking, Shvedov proved Theorem 1.6 in the case of $K_{i}$ being finite. However, the proof in [Ku] easily modifies to yield the general case.

\section{UNIVERSAL CELL-LIKE MAPS}

2.1. Lemma. Suppose $f: X \rightarrow Y$ is a proper map of separable metrizable spaces and $K$ is a countable $C W$ complex. Then, the set

$$
Z=\left\{y \in Y \mid\left[f^{-1}(y), K\right]=0\right\}
$$

is a $G_{\delta}$-set in $Y$.

Proof. Choose a metrizable compactification $X^{\prime}$ of $X$, and choose a sequence $\left\{F_{i}\right\}_{i \geq 1}$ of closed subsets of $X^{\prime}$ such that for any closed subset $A$ of $X^{\prime}$ and for any neighborhood $U$ of $A$ there exists $k$ with $A \subset$ int $F_{k} \subset F_{k} \subset U$. For each $i$ choose a sequence $g_{i, k}: F_{i} \rightarrow K, k \geq 1$, of maps such that for any map $g: F_{i} \rightarrow K$ there is $k$ such that $g \simeq g_{i, k}$. Put

$$
\begin{aligned}
G_{i}=\left\{y \in Y: f^{-1}(y) \subset \operatorname{int} F_{i} \text { and } g_{i, k} \mid f^{-1}(y) \simeq\right. \text { const } \\
\text { for all } \left.k \text { or } f^{-1}(y) \cap\left(X^{\prime}-\operatorname{int} F_{i}\right) \neq \varnothing\right\} .
\end{aligned}
$$

Then, $Z=\bigcap G_{i}$. Since each set

$$
\left\{y \in Y: f^{-1}(y) \subset \text { int } F_{i} \text { and } g_{i, k} \mid f^{-1}(y) \simeq \text { const }\right\}
$$

is open and the set

$$
\left\{y \in Y: f^{-1}(y) \cap\left(X^{\prime}-\operatorname{int} F_{i}\right) \neq \varnothing\right\}
$$

is closed, each $G_{i}$ is a $G_{\delta}$-set.

2.2. Theorem. Suppose $n \leq \infty$. There is a cell-like map $f: X \rightarrow Y$ of complete and separable metric spaces and a map $v: Y \rightarrow Q$ from $Y$ to the Hilbert cube $Q$ such that the following conditions are satisfied:

(a) $\operatorname{dim} X \leq n$,

(b) for any cell-like map $f^{\prime}: X^{\prime} \rightarrow Y^{\prime}$ of separable metric spaces with $\operatorname{dim} X^{\prime} \leq n$ and $Y^{\prime} \subset Q$ there exist embeddings $i: Y^{\prime} \rightarrow Y$ and $j: X^{\prime} \rightarrow$ $f^{-1}(Y)$ such that $f j=i f^{\prime}, \mathrm{id}=v i$, and $f^{-1}\left(i\left(Y^{\prime}\right)\right)=j\left(X^{\prime}\right)$.

Proof. Let $\alpha: M \rightarrow Q$ be a map of compacta such that $\operatorname{dim} M \leq n$, and for any map $g: Z \rightarrow Q, Z$ being $n$-dimensional and separable, there is an embedding $i: Z \rightarrow M$ such that $\alpha i=g$ (see Corollary 3.4). Let $\beta: C \rightarrow 2^{M}$ be a surjective map from the Cantor set onto the hyperspace $2^{M}$ of $M$. Consider the subspace $A=\bigcup\{\{c\} \times \beta(c) \mid c \in C\}$ of $C \times M$. Define $\mu: A \rightarrow C \times Q$ by $\mu(c, x)=(c, \alpha(x))$. Let $Y$ be the set of all $y \in C \times Q$ such that $\mu^{-1}(y)$ is celllike. By Lemma $2.1, Y$ is a $G_{\delta}$-set in $C \times Q$; hence, $Y$ is completely metrizable. Let $X=\mu^{-1}(Y), f: X \rightarrow Y$ be the restriction of $\mu$, and $v: Y \rightarrow Q$ be the projection onto the last coordinate. Suppose $f^{\prime}: X^{\prime} \rightarrow Y^{\prime}$ is a cell-like map of separable metric spaces with $\operatorname{dim} X^{\prime} \leq n$ and $Y^{\prime} \subset Q$. Choose an embedding $i^{\prime}: X^{\prime} \rightarrow M$ such that $\alpha i^{\prime}=f^{\prime}$. Without loss of generality we may assume that $i^{\prime}=$ id (i.e., $\left.X^{\prime} \subset M\right)$. Choose $c \in C$ with $\operatorname{cl}\left(X^{\prime}\right)=\beta(c)$. Notice that $\mu\left(\{c\} \times X^{\prime}\right) \subset Y$. Indeed, suppose $\alpha(x)=y \in Y^{\prime}$ and $x \in \operatorname{cl}\left(X^{\prime}\right)$. Choose a sequence $x_{n} \in X^{\prime}$ converging to $x$ so that $\alpha\left(x_{n}\right) \rightarrow y$. If $x \notin X^{\prime}$, then the set 
$\left\{x_{n}\right\}_{n \geq 1}$ is closed in $X^{\prime}$. Hence, $\left\{\alpha\left(x_{n}\right)\right\}_{n \geq 1}$ is closed in $Y^{\prime}$ and $y=\alpha\left(x_{k}\right)$ for infinitely many $k$. Thus, $x \in\left(f^{\prime}\right)^{-1}(y)$ and $x \in X^{\prime}$.

Finally, $j: X^{\prime} \rightarrow X$ is defined by $j(x)=(c, x)$ and $i: Y^{\prime} \rightarrow Y$ is defined by $i(y)=(c, y)$.

2.3. Corollary. Suppose $n<\infty$. There is a complete and separable metric space $Z$ such that $\operatorname{dim}_{\mathrm{Z}} Z \leq n$ and any (complete) separable metric space $Y^{\prime}$ with $\operatorname{dim}_{\mathrm{Z}} Y^{\prime} \leq n$ embeds as a (closed) subset of $Y$.

Proof. Choose $v: Y \rightarrow Q$ as in Theorem 2.2. Put $Z=v^{-1}(s)$, where $s$ is the pseudo-interior of $Q$.

\section{UNIVERSAL MAPS}

Theorem 1.3 was deduced in $\left[\mathrm{Dy}_{1}\right]$ from the following theorem:

3.1. Theorem. Suppose $u: K \rightarrow L$ is a map from a countable $C W$ complex $K$ to a compact metrizable space $L$. Given a compactum $X$ there is a compactum $X^{\prime}$ and a map $\pi: X^{\prime} \rightarrow X$ with the following properties:

(a) Given $f: Y \rightarrow X, K \in A E(Y)$, there is $f^{\prime}: Y \rightarrow X^{\prime}$ with $f=\pi f^{\prime}$,

(b) Given $g: C \rightarrow K, C$ closed in $X^{\prime}$, there is $g^{\prime}: X^{\prime} \rightarrow L$ with $g^{\prime} \mid C$ homotopic to $\mathrm{ug}$.

This result generalized Shvedov's Theorem (dealing with existence of compactifications) and at the same time it produced universal maps which led to the existence of universal spaces. The purpose of this section is to separate the issue of universal maps from the issue of compactifications and to prove a result which, together with Shvedov's Theorem, implies Theorem 1.3.

3.2. Theorem. Suppose $K$ is a countable $C W$ complex. There is a map $\pi$ : $M \rightarrow Q$ of a completely metrizable space $M$ to the Hilbert cube $Q$ such that $K \in A E(M)$ and for any map $g: X \rightarrow Q, X$ compact metrizable, and $K \in$ $A E(X)$, there is an embedding $i: X \rightarrow M$ with $\pi i=g$.

Proof. Consider the subset $A$ of the hyperspace $2 Q \times Q$ of $Q \times Q$ consisting of compacta $X$ with $K \in A E(X)$. By Theorem 3.1 of [D-R], $A$ is a dense $G_{\delta^{-}}$ subset of $2^{Q \times Q}$. Choose a surjective map $\alpha: C \rightarrow 2^{Q \times Q}$ from the Cantor set $C$ onto $2^{Q \times Q}$. Notice that $B=\alpha^{-1}(A)$ is a $G_{\delta}$-set in $C$, hence $B$ is completely metrizable. Let $M=\bigcup\{\{p\} \times \alpha(p) \mid p \in B\} \subset B \times Q \times Q$. Let $\pi: M \rightarrow Q$ be the projection onto the last coordinate. Given $g: X \rightarrow Q, X \subset Q$ compact and $K \in A E(X)$, define $g^{\prime}: X \rightarrow Q \times Q$ by $g^{\prime}(x)=(x, g(x)), x \in X$. Let $c \in C$ satisfy $\alpha(c)=g^{\prime}(X)$. Define $i: X \rightarrow M$ by $i(x)=(c, x, g(x))$, $x \in X$. Then, $\pi i=g$.

It remains to show that $K \in A E(M)$. Notice that the projection $p: M \rightarrow B$ is a closed map. Suppose $h: D \rightarrow K$ and $D$ is closed in $M$. For every $b \in B$ there is an extension $h_{b}: C \cup p^{-1}\left(U_{b}\right) \rightarrow K$ of $h$, where $U_{b}$ is an open-closed neighborhood of $b$ in $B$. Choose a countable subset $\{b(n)\}_{n \geq 1}$ of $B$ such that $\bigcup U_{b(n)}=B$. Now, $h^{\prime}: M \rightarrow K$ defined by $h^{\prime}(x)=h_{b(n)}(x)$, where $n$ is the smallest integer so that $x \in U_{b(n)}$, is an extension of $h$.

3.3. Corollary. Suppose $K$ is a countable $C W$ complex such that any separable metrizable space $Y$ with $K \in A E(Y)$ admits a metrizable compactification $Y^{\prime}$ with $K \in A E\left(Y^{\prime}\right)$. Then, there is a universal compactum $X$ in the class of all compacta $Y$ such that $K \in A E(Y)$. 
Proof. Choose $M$ as in Theorem 3.2, and let $X$ be a metrizable compactification of $M$ such that $K \in A E(X)$.

3.4. Corollary. Suppose $K$ is a countable $C W$ complex such that any metrizable compactification $c(X)$ of a separable space $X$ with $K \in A E(X)$ admits a metrizable compactification $c^{\prime}(X) \geq c(X)$ (i.e., there is a map $h: c^{\prime}(X) \rightarrow c(X)$, $h \mid X=\mathrm{id})$ with $K \in A E\left(c^{\prime}(X)\right)$. Then, there is a map $s: Z \rightarrow Q$ of a compactum $Z$ to the Hilbert cube $Q$ such that $K \in A E(Z)$ and for any map $g: X \rightarrow Q, X$ separable metrizable, and $K \in A E(X)$, there is an embedding $i: X \rightarrow Z$ with $s i=g$.

Proof. Choose $M$ as in Theorem 3.2. We may assume $M \subset Q$. Let $c(M)$ be the closure in $Q \times Q$ of the image of $M$ under the map $x \rightarrow(x, \pi(x))$. Let $Z$ be a metrizable compactification of $M$ such that $K \in A E(Z)$ and there is a map $h: Z \rightarrow c(M)$ with $h(x)=(x, \pi(x))$ for $x \in M$. Define $s$ as the composition of $h$ and the projection of $Q \times Q$ onto the last coordinate.

Remark. In case of $K=S^{n}$ one can construct maps with much stronger properties than those in Corollary 3.4 (see $\left[\mathrm{Dr}_{4}\right]$ ). Our next result shows that the hypotheses of Corollary 3.4 cannot be omitted and that the space $M$ in Theorem 3.2 cannot be compact, in general.

3.5. Theorem. For any map $s: Z \rightarrow Q$ of a compactum $Z$ to the Hilbert cube $Q$ such that $\operatorname{dim}_{\mathrm{Z}} Z \leq n$ there is a compactum $X \subset Q$ such that $\operatorname{dim}_{\mathrm{Z}} X \leq n$ and the inclusion $X \hookrightarrow Q$ does not lift to $Z$.

Proof. Choose a $\mathrm{CW}$ complex $K$ being a $K(\mathrm{Z}, n)$ and having finite skeleta. Let $X_{k} \subset Q$ be compacta as in [D-W]. Thus, $X_{k}$ has the following properties:

(a) $\operatorname{dim}_{\mathbf{Z}} X_{k} \leq n$ for each $k$,

(b) each $X_{k}$ contains the same copy of $S^{n}$,

(c) the inclusion $S^{n} \hookrightarrow K$ cannot be extended over $X_{k}$ so that the image of the extension is contained in the $k$-skeleton $K^{(k)}$ of $K$.

Suppose $s: Z \rightarrow Q$ exists, and choose an extension $g: Z \rightarrow K$ of $s^{-1}\left(S^{n}\right) \rightarrow S^{n} \hookrightarrow K$. There is $k \geq 1$ such that $g(Z) \subset K^{(k)}$ which demonstrates that $X_{k} \hookrightarrow Q$ cannot be lifted to $Z$.

Remark. In view of Theorem 3.5 and its proof one is inclined to believe that there is a stronger connection between existence of compactifications and the existence of universal spaces than that of Corollary 3.3. We conjecture that universal spaces for integral cohomological dimension do not exist based on the fact that, in general, compactifications preserving cohomological dimension do not exist (see $\left[\mathrm{Dr}_{2}\right],[\mathrm{D}-\mathrm{W}]$ and $\left.\left[\mathrm{Dy}_{2}\right]\right)$.

\section{REFERENCES}

[Ch] A. Chigogidze, A note on cohomological dimension, preprint.

[D-R] T. Dobrowolski and L. Rubin, The hyperspaces of infinite-dimensional compacta for covering and cohomological dimension are homeomorphic, Pacific J. Math. 163 (1994), 15-39.

[Dr $\mathrm{Dr}_{1}$ A. N. Dranishnikov, Homological dimension theory, Uspekhi Mat. Nauk 43 (1988), 11-55. (Russian)

[Dr $\mathrm{Dr}_{2} \longrightarrow$, Cohomological dimension is not preserved by Stone-Čech compactification, C. $\mathbf{R}$. Bulgar. Acad. Sci. 41 (1988), 9-10. (Russian) 
[Dr 3 _ Cohomological dimension theory, Lectures at University of Tennessee, 1989.

[Dr $\left.{ }_{4}\right]-$ Universal Menger compacta and universal mappings, Math. USSR-Sb. 57 (1987), 131-149.

[D-S] J. Dydak and J. Segal, Shape theory: An introduction, Lecture Notes in Math., vol. 688, Springer-Verlag, New York, 1978, pp. 1-150.

[D-W] J. Dydak and J. J. Walsh, Spaces without cohomological dimension preserving compactifcations, Proc. Amer. Math. Soc. 113 (1991), 1155-1162.

[Dy $\mathrm{D}_{1}$ J. Dydak, Cohomological dimension and metrizable spaces II, preprint.

[Dy $\left.{ }_{2}\right]-$ Compactifications and cohomological dimension, Topology Appl. 50 (1993), 1-10.

[Hu] S. T. Hu, Theory of retracts, Wayne State Univ. Press, Detroit, MI, 1965.

[Ku] W. I. Kuzminov, Homological dimension theory, Russian Math. Surveys 23 (1968), 1-45.

[Ru] L. R. Rubin, Universal metrizable compacta for integral cohomological dimension, preprint.

[R-S] L. R. Rubin and P. J. Schapiro, Cell-like maps onto non-compact spaces of finite cohomological dimension, Topology Appl. 27 (1987), 221-244.

[Sp] E. Spanier, Algebraic topology, McGraw-Hill, New York, 1966.

[Wa] J. J. Walsh, Dimension, cohomological dimension, and cell-like mappings, Lecture Notes in Math., vol. 870, Springer-Verlag, New York, 1981, pp. 105-118.

[We] J. West, Open problems in infinite dimensional topology, Open Problems in Topology, North-Holland, Amsterdam, 1990.

[Wh] George W. Whitehead, Elements of homotopy theory, Springer-Verlag, New York, 1978.

Department of Mathematics, University of Tennessee, Knoxville, Tennessee 37996

E-mail address: dydak@math.utk.edu

Department of Mathematics, Knoxville College, Knoxville, Tennessee 37921

E-mail address: mogilski@math.utk.edu 